of nodal peripheral T-cell lymphoma demonstrates a molecular link between angioimmunoblastic T-cell lymphoma (AITL) and follicular helper T (TFH) cells. Blood. 2007;109(11):4952-4963.

6. Piccaluga PP, Agostinelli C, Califano A, et al. Gene expression analysis of peripheral T cell lymphoma, unspecified, reveals distinct profiles and new potential therapeutic targets. J Clin Invest. 2007;117(3):823-834

7. Cortes JR, Ambesi-Impiombato A, Couronne L, et al. RHOA G17V Induces T Follicular Helper Cell Specification and Promotes Lymphomagenesis. Cancer Cell. 2018;33(2):259-273.e7.
8. Heavican TB, Bouska A, Yu J, et al. Genetic drivers of oncogenic pathways in molecular subgroups of peripheral T-cell lymphoma. Blood. 2019;133(15):1664-1676.

9. Pedersen MB, Hamilton-Dutoit SJ, Bendix K, et al. DUSP22 and TP63 rearrangements predict outcome of ALK-negative anaplastic large cell lymphoma: a Danish cohort study. Blood. 2017;130(4):554-557.

10. Drieux F, Ruminy P, Abdel-Sater A, et al. Defining signatures of peripheral T-celllymphoma with a targeted 20-marker gene expression profiling assay.. Haematologica. 2020;105(6):1582-1592.

\title{
PIKing the next therapeutic target in multiple myeloma
}

\author{
Jessica L. Caro and Faith E. Davies \\ Perlmutter Cancer Center, NYU Langone Health, New York, NY, USA \\ E-mail: FAITH E DAVIES - faith.davies@nyulangone.org
}

doi:10.3324/haematol.2020.248971

$\Lambda$ rguably the most transformative myeloma therapies to date have been those which target essential processes involved in plasma cell function. Although their mechanism of action may not have been entirely obvious when first introduced, it has now become clear from cell-based studies that these therapies target protein degradation via the ubiquitin proteasome pathway, a critical process for plasma cell survival. Examples include proteasome inhibitors (such as bortezomib, carfilzomib, and ixazomib) and the immunomodulatory drugs (such as lenalidomide and pomalidomide), which inhibit the CUL4 E3 ubiquitin ligase, cereblon. Both drug classes are the 'go to' choices in current myeloma treatment. ${ }^{1}$ It therefore comes as no surprise that the search for other therapies targeting protein degradation pathways continues.

The key function of a normal plasma cell is to produce immunoglobulins. Studies have shown that myeloma plasma cells, which produce large quantities of $\mathrm{M}$ protein, are highly dependent on the multiple pathways that enable a cell to handle excess unfolded or misfolded proteins. Over the last 10 years, cancer researchers have explored many of these pathways with a view to therapeutic exploitation. The rationale is that inhibition of these pathways leads to a build up of unwanted or misfolded proteins, the induction of cellular stress, and ultimately to cancer cell death. Such pathways include not only the ubiquitin proteasome pathway but also the heat shock protein pathway, autophagy pathway, unfolded protein response pathway, and pathways involving lysosomes and aggresomes. ${ }^{2}$ However, translating in vitro findings into clinical success has been difficult. It has become clear that some cancer types are dependent on one pathway more than others, the pathways are interlinked, and the crosstalk between pathways enables the development of both primary and drug-induced mechanisms of resistance.

In this edition of Haematologica, Bonolo de Campos et al. describe a promising new approach for myeloma therapy by perturbing the autophagy and lysosome pathways. ${ }^{3}$ In autophagy, misfolded and aggregated proteins are sequestered in double-membraned vesicles called autophagosomes that eventually fuse with lysosomes for digestion and recycling. Previous studies have shown that myeloma cells require tight regulation of the autophagy pathway for cell survival, and genetic or therapeutic manipulation of this pathway induces growth inhibition and/or cell death. ${ }^{2,4,5}$

Phosphatidylinositol-3-phosphate 5 kinase (PIKfyve) is a phosphoinositide kinase with many diverse functions within the cell, including the generation of phosphorylated substrates critical to the regulation of autophagy. Inhibition of PIKfyve using the selective inhibitor apilimod has previously been investigated as a potential therapeutic approach for both inflammatory diseases and nonHodgkin lymphoma. ${ }^{7}$ Using an unbiased chemical screen, Bonolo de Campos et al. identified APY0201 and examined its activity along with that of apilimod and another novel PIKfyve inhibitor YM201636 in 25 human myeloma cell lines and 100 ex-vivo patient-derived samples. They confirmed dose-dependent inhibition of cell viability in all myeloma cell lines, with APY0201 being the most potent PIKfyve inhibitor. They additionally observed dosedependent sensitivities in $40 \%$ of ex-vivo patient-derived samples with APY0201. Mechanistic experiments suggested that exposure to APY0201 resulted in activation of the transcription factor EB (TFEB) leading to upregulation of autophagosome and lysosomal biogenesis. Exposure also disrupted lysosomal function leading to alterations in autophagic flux and a vacuolization phenotype.

As myeloma is a genetically and biologically heterogeneous disease, it is critical to identify which patients would benefit most from a new therapy. The prime example of the need for such an approach is venetoclax, a Bcl-2 inhibitor, which has been shown to be particularly efficacious in patients harboring a $\mathrm{t}(11 ; 14)$ translocation. ${ }^{8}$ Although targeting a pathway central to plasma cell survival should theoretically result in universal myeloma cell death, it has become clear that the genetic background of the cell influences response to therapy.9 For instance, whereas patients with a $t(14 ; 16)$ translocation tend to respond poorly to proteasome inhibitors, these therapies may be able to overcome some of the adverse outcome associated with the $t(4 ; 14)$ subgroup. ${ }^{10}$ Therefore, trying to incorporate genetic information into therapeutic decisionmaking may allow us to optimize treatment choices and response rates and to provide long-lasting remissions. Importantly, the authors have tried to assess this in their 
study using data from the patients' samples. The activity of APY0201 was highest in patient-derived samples with hyperdiploidy (trisomies with one or more odd-numbered chromosomes) and lowest in patients' samples with a $\mathrm{t}(11 ; 14)$ translocation. In addition, ex-vivo samples with high TFEB levels were sensitive to APY0201. High TFEB levels have been associated with increased autophagic flux suggesting that autophagic flux may be directly related to PIKfyve inhibition. These preliminary results may suggest patient populations that could be enriched for in a future clinical trial.

In conclusion, Bonolo de Campos et al. provide exciting data to support the ongoing investigation of therapeutically manipulating targets specific to plasma cell function, particularly protein handling in myeloma. ${ }^{2}$ Although the finer details of the actual mechanisms may differ somewhat between multiple myeloma and non-Hodgkin lymphoma, data from this study and those performed in nonHodgkin lymphoma provide compelling evidence for the role of PIKfyve inhibition in inducing cell death, with changes seen in the autophagy and lysosomal pathways. Notably, this study demonstrates the importance of the inherent genetic differences in myeloma biology and the potential role of PIKfyve inhibitors in targeting a distinct group of genetically defined myeloma to continue this era of personalized medicine.

\section{References}

1. Gay F, Engelhardt M, Terpos E, et al. From transplant to novel cellular therapies in multiple myeloma: European Myeloma Network guidelines and future perspectives. Haematologica. 2018;103(2):197-211.

2. Aronson LI, Davies FE. DangER: protein ovERload. Targeting protein degradation to treat myeloma. Haematologica. 2012;97(8):11191130.

3. Bonolo De Campos C, Zhu YX, Sepetov N, et al. Identification of PIKfyve kinase as a target in multiple myeloma. Haematologica. 2020;105(6):1641-1649.

4. Hoang B, Benavides A, Shi Y, Frost P, Lichtenstein A. Effect of autophagy on multiple myeloma cell viability. Mol Cancer Ther 2009;8(7):1974-1984.

5. Aronson LI, Davenport EL, Mirabella F, Morgan GJ, Davies FE. Understanding the interplay between the proteasome pathway and autophagy in response to dual PI3K/mTOR inhibition in myeloma cells is essential for their effective clinical application. Leukemia. 2013;27(12):2397-2403

6. Hessvik NP, Øverbye A, Brech A, et al. PIKfyve inhibition increases exosome release and induces secretory autophagy. Cell Mol Life Sci. 2016;73(24):4717-4737.

7. Gayle S, Landrette S, Beeharry N, et al. Identification of apilimod as a first-in-class PIKfyve kinase inhibitor for treatment of B-cell nonHodgkin lymphoma. Blood. 2017;129(13):1768-1778.

8. Kumar S, Kaufman JL, Gasparetto C, et al. Efficacy of venetoclax as targeted therapy for relapsed/refractory t(11;14) multiple myeloma. Blood. 2017;130(22):2401-2409.

9. Pawlyn C, Davies FE. Toward personalized treatment in multiple myeloma based on molecular characteristics. Blood. 2019 14;133(7):660-675.

10. Qiang YW, Ye S, Chen Y, et al. MAF protein mediates innate resistance to proteasome inhibition therapy in multiple myeloma. Blood. 2016;128(25):2919-2930.

\title{
The increasing complexity of the management of core-binding factor acute myeloid leukemia
}

\section{Mark R. Litzow}

\author{
Division of Hematology and Transplant Center, Mayo Clinic Rochester, Rochester, MN, USA \\ E-mail: MARK R. LITZOW - litzow.mark@mayo.edu
}

doi:10.3324/haematol.2020.249110

T he core binding factor (CBF) acute myeloid leukemias characterized by the $\mathrm{t}(8 ; 21)$ and $\operatorname{inv}(16)(\mathrm{p} 13 \mathrm{q} 22) / \mathrm{t}(16 ; 16)(\mathrm{p} 13 ; \mathrm{q} 22) \quad$ cytogenetic abnormalities have long been known to prognostically represent more favorable subcategories of acute myeloid leukemia (AML). These translocations are characterized by the presence of the RUNX1-RUNX1T1 (AML1-ETO) and $C B F B-M Y H 11$ fusion transcripts, respectively. In fact, the $t(8 ; 21)$ was the first cytogenetic abnormality identified in AML in 1973. ${ }^{1}$ These CBF-AML subtypes have continued to remain in the favorable risk category in multiple classification systems up to the current time based on their high rate of achievement of complete remission with induction chemotherapy and their relatively low relapse rate. ${ }^{2}$ Clinical trials over the years have demonstrated that these two CBF-AML subtypes are particularly responsive to high doses of cytarabine utilized in consolidation regimens. Addition of the immunoconjugate drug, gemtuzumab ozogamicin, to induction chemotherapy further reduces the risk of relapse and improves overall survival in patients with CBF-AML. ${ }^{3}$ The favorable results of chemotherapy in patients with CBF-AML have led to the widely accepted practice not to perform allo- geneic blood or marrow transplant (alloBMT) in these patients who achieve first remission. This is in contrast to patients with AML with intermediate risk or unfavorable risk features where allogeneic blood or marrow transplant in first remission is a widely accepted practice.

However, the two subtypes of CBF-AML are not the same in all respects. Studies going back 15 years or more have pointed out the difference between these two subtypes. ${ }^{4}$ Use of next-generation sequencing (NGS) and identification of additional gene mutations in patients with AML have begun to further define differences between the two. One of the first mutational abnormalities found in subsets of patients with CBF-AML were cKIT mutations. The $c-K I T$ mutation has been suggested to be associated with a poorer prognosis in CBF-AML patients but, here again, this mutation seems to have less of a prognostic impact in patients with inv(16) compared to those with $\mathrm{t}(8 ; 21) .{ }^{5} \mathrm{NGS}$ studies, which are now widely utilized to assess prognosis in many subtypes of AML, have been applied to patients with CBF-AML. Multiple mutations in addition to c-KIT have been identified, including genes activating tyrosine kinase signaling, such as N/KRAS and FLT3. Mutations in genes that regulate 